\title{
De dependencias y prohibiciones alcohólicas: un análisis previo y durante la covid-19 en Yucatán*
}

\author{
From dependency to alcoholic prohibition: \\ A preliminary and ongoing analysis during coviD-19 in Yucatán
}

\author{
SERGIO A. MORENO CABRERA**
}

\begin{abstract}
We propose analyzing the link between social knowledge about the consequences of alcohol consumption, with the alcoholic prohibitions implemented in Yucatán during COVID-19; this ethnographic study took place in the state municipality of Cuzamá from 2016 to 2017 from a critical medical anthropological stance with a relational approach. The information on prohibitions and their effects was documented during 2020. This ethnographical study shows a culture ambivalent toward alcohol and its health consequences, along with a preference for collective consumption and a dependence that exceeds the biomedical-individual criteria. Alcoholic prohibitions confirm the tension between positivity and negativity, between the ideal and the reality of consumption. Despite the questions that remain, such as going further in depth regarding "collective dependence", we consider this article to be an important contribution due to its approaches, and a valuable input for the analysis of public policies for alcohol consumption in Mexico.
\end{abstract}

Key words: critical medical anthropology, alcohol consumption, alcohol and ambivalence, collective dependence, COVID-19

\section{Resumen}

Se propone analizar la vinculación entre los saberes sociales sobre las consecuencias del consumo de alcoholy las prohibiciones alcohólicas implementadas en Yucatán en tiempos de la Covid-19; la etnografía se desarrolló durante 2016-201 7 en el municipio de Cuzamá desde la antropología médica crítica y con un enfoque relacional, y la información sobre las prohibiciones y sus efectos fue documental y se obtuvo durante 2020. La etnografía muestra una ambivalencia cultural respecto del alcohol y sus consecuencias sanitarias, junto con la preferencia por el consumo colectivo y una dependencia que supera los criterios biomédico-individuales. Las prohibiciones alcohólicas confirman la tensión entre sus cualidades positivas y negativas, entre lo ideal y lo real, de este consumo. Pese a las preguntas que quedan pendientes -como profundizar en la dependencia colectiva-, consideramos que el artículo es una contribución al tema debido a sus planteamientos, y que es un valioso insumo para el análisis de las políticas públicas sobre el consumo de alcohol en México.

Palabras clave: antropología médica crítica, consumo de alcohol, alcohol y ambivalencia, dependencia colectiva, COVID-19

\footnotetext{
* Artículo recibido el $02 / 10 / 20$ y aceptado el 20/01/21.

** Consultor independiente; docente en Universidad Marista de Mérida <sergio.moreno.yuc@gmail.com>. oRcID: https:// orcid.org/0000-0002-2611-0596
} 


\section{Introducción}

$\mathrm{E}$ 1 análisis sociocultural de los procesos de salud/ enfermedad/atención-prevención (s/e/a-p) nos ha permitido comprender las formas en las que los sujetos y grupos sociales entienden y atienden las enfermedades, los padecimientos y la muerte, pero también los aspectos estructurales que les subyacen; más aún, los procesos de s/e/a-p llegan a constituirse, en palabras de Berlinguer (cit. en Menéndez Spina, 2015), en "espías" de las contradicciones de todo sistema social, sacando a la luz sus razones y sinrazones.

Durante la contingencia sanitaria por COVID-19, uno de los procesos de s/e/a-p más debatidos en Yucatán -y en México- fue la prohibición y regulación del consumo de alcohol. En aras de proteger la salud pública de la población, la autoridad estatal impulsó diversas medidas, entre ellas la prohibición de la venta de bebidas alcohólicas, llamada de manera coloquial "ley seca”. Después de muchas críticas se autorizó la reactivación parcial de su venta, seguida de un nuevo periodo de prohibición y de una nueva reactivación. Los argumentos en favor y en contra de parte de diferentes sectores de la población, tanto gubernamentales como de la ciudadanía en general, permiten analizar las representaciones sociales locales sobre este consumo y sobre sus variadas consecuencias.

Entre los beneficios de la prohibición aparece la reducción de las situaciones sociales de consumo colectivo y con ello el riesgo de contagios, favoreciendo incluso la disminución de los casos de intoxicación aguda por alcohol en todo el estado. No obstante, entre los perjuicios de esta medida destaca el aumento de la venta y consumo clandestino de estas bebidas (legales o adulteradas), derivando en la muerte de casi una veintena de personas durante el primer trimestre de la pandemia.

El apoyo y rechazo social expresado simultáneamente ante estas prohibiciones alcohólicas ${ }^{1}$ en Yucatán en tiempos de la coviD- 19 muestran una aparente contradicción sobre lo que se piensa y lo que se hace respecto de lo "bueno" y lo "malo" del alcohol, sobre quiénes "saben" o "no saben" beber, y sobre quiénes pueden o no pueden "controlarse", así como sobre la paradoja del cuidado de la salud de la población. De tal suerte, este trabajo busca problematizar ${ }^{2}$ la prohibición y la dependencia alcohólicas en Yucatán desde una mirada sociocultural, que sume a la comprensión de este consumo y del proceso de alcoholización ${ }^{3}$ en general.

El artículo se organiza en tres apartados: el primero presenta una síntesis de las prohibiciones gubernamentales emitidas en 2020 en el marco de la pandemia, analizando los "olvidos", efectos y ambivalencia que las caracterizó; el segundo y tercer apartados ofrecen información etnográfica prepandemia, que confirma, por un lado, que los conjuntos sociales reconocen de un modo ambivalente lo negativo y lo positivo de este consumo en su salud integral y, por otro, evidencia una especie de "dependencia" al consumo colectivo de alcohol, que parece confirmarse en tiempos de la COVID-19, y que no puede entenderse sólo desde la perspectiva biomédica y en los términos de la adicción.

Como nota metodológica valga señalar que la información etnográfica presentada en el segundo apartado se desprende de mi trabajo de investigación doctoral (Moreno Cabrera, 2019), realizado entre 2016 y 2017 en Cuzamá, municipio céntrico de Yucatán, México, ubicado en la exzona henequenera del estado, a 48 kilómetros de la ciudad capital (Mérida).

Dicha investigación se llevó a cabo desde la antropología médica crítica y con un enfoque relacional (Menéndez Spina, 2009a). A través de conversaciones informales, entrevistas y de la observación participante se describieron y analizaron los saberes sociales ${ }^{4}$ de hombres y mujeres de 25 a 40 años de edad sobre las consecuencias positivas, negativas y ambivalentes del consumo de alcohol en su salud, recursos económicos y relaciones sociales.

\section{Prohibición y covid-19: "gobiernos" de alcohol de ayer y hoy}

Después de que la Organización Mundial de la Salud hiciera la declaración mundial de pandemia por el virus SARS-CoV-2 en marzo de 2020, el gobierno del estado de Yucatán decretó el 9 de abril la prohibición de la venta de bebidas alcohólicas en todo el territorio

\footnotetext{
1 Las nombro en plural, pues no fue una sola acción gubernamental sino varias (decretos).

2 Proceso de distanciamiento de un fenómeno o experiencia para convertirlo en objeto de análisis y poder así "hacerle preguntas" y comprenderlo con mayor profundidad (Bourdieu, Chamboredon y Passeron, 2002, y seminarios presenciales de Menéndez Spina en 2016).

3 Procesos económico-políticos y socioculturales que operan en una situación histórica determinada, y que configuran las características dominantes del uso y consumo de alcohol, así como su no uso y no consumo, por parte de sujetos y conjuntos sociales. El proceso de alcoholización expresa aspectos estructurales sobre los que descansa, y desde donde debe ser analizado, el consumo de alcohol, el alcoholismo y el complejo alcohólico (Menéndez y Di Pardo, 1996).

4 Los saberes integran las representaciones y prácticas de los sujetos sociales, esto es, lo que creen y dicen sobre el mundo y sus relaciones, pero también lo que hacen. Pero los saberes sociales no son siempre congruentes, continuos ni
} 
estatal, como parte de una serie de medidas sanitarias de "protección de la salud" de las familias yucatecas, incluyendo en sus intenciones la prevención de la violencia familiar.

El cuadro 1 sintetiza las fechas y decretos gubernamentales emitidos del 9 de abril hasta finales de 2020, así como una breve mención de los argumentos utilizados para justificar la implementación, extensión, suspensión o aplicación parcial de la prohibición de venta de bebidas alcohólicas en Yucatán en razón de la pandemia por COVID-19.

Como se puede leer, entre las consideraciones para implementar estas medidas aparecen motivos sanitarios como la prevención de contagios por COVID-19 o la alerta por la llegada de huracanes, pero también el reconocimiento de los beneficios económicos de esta actividad, y explicaciones imprecisas como la vinculación entre el alcohol y la violencia.

Mi análisis no pone en duda la necesidad de una respuesta gubernamental ante una emergencia sanitaria de la magnitud como la que ahora enfrentamos, más aún, la considero esencial. Lo que cuestiono, e invito al diálogo, es la "racionalidad" gubernamental detrás de los argumentos prohibicionistas que poco parecen conocer y/o comprender las características socioculturales -o algunas de ellas- del consumo de alcohol en Yucatán en tanto proceso de s/e/a-p.

Una de estas racionalidades es la aparente certeza científica y gubernamental que yace en dichos decretos y que sugiere que de seguirse ad pedem litterae garantizarán la protección de la salud y el bienestar de la población; no obstante, lo que resultó fueron una serie de ensayos y errores que derivaron en ajustes y derogaciones de estos decretos gubernamentales.

Quizá ayude recordar que estas prohibiciones han mostrado a lo largo de la historia -propia y ajena- ${ }^{5}$ que poco o nada han logrado respecto del consumo y abuso de alcohol, en parte porque la ciencia biomédica tampoco escapa de las representaciones morales e ideológicas -ni a las relaciones de poder-que subyacen a todo proceso de s/e/a-p (Menéndez Spina, 2020), y en parte porque la propia población, a pesar de tener conocimiento de los aspectos negativos de esta ingesta, continúa bebiendo y experimentando su lado positivo (Heath, 2012; Moreno Cabrera, 2020a).

Uno de los primeros esfuerzos por regular y controlar el consumo de alcohol, y a los consumidores yucatecos/as, se remonta a finales del siglo xIX y principios del $x x$ en los tiempos del porfiriato, ${ }^{6}$ y fue impulsada por un movimiento sociopolítico de temperancia, dirigido en especial a la población indígena y campesina que era explotada en la industria henequenera, y sobre la cual se instauraron una serie de políticas y "gobiernos" en contra de este "vicio", desde un discurso de “orden y progreso” (Márquez Osuna, 2014).

Angélica Márquez Osuna nombra gobiernos del alcohol al "conglomerado de circunstancias inestables, movimientos heterogéneos y confluencia de saberes que dieron dirección a diversas técnicas y estrategias para controlar el consumo de esta sustancia" (2014: 6), encontrando en su revisión no una política gubernamental clara y bien definida, sino una de múltiples formas, ensayos y errores.

Como ejemplo de ello cita los esfuerzos llevados a cabo en ese entonces para definir el consumo "excesivo" e "inmoderado", enunciándose como enemigos del desarrolloy progreso, pero cuya medición se caracterizó por ambigüedades e imprecisiones y no por certezas médicas o políticas. Más todavía, dice la autora, el consumo inmoderado estaba sujeto a razones de género y de "raza" y no a criterios de cantidad, desplazándose así al terreno de lo moral (Márquez Osuna, 2014).

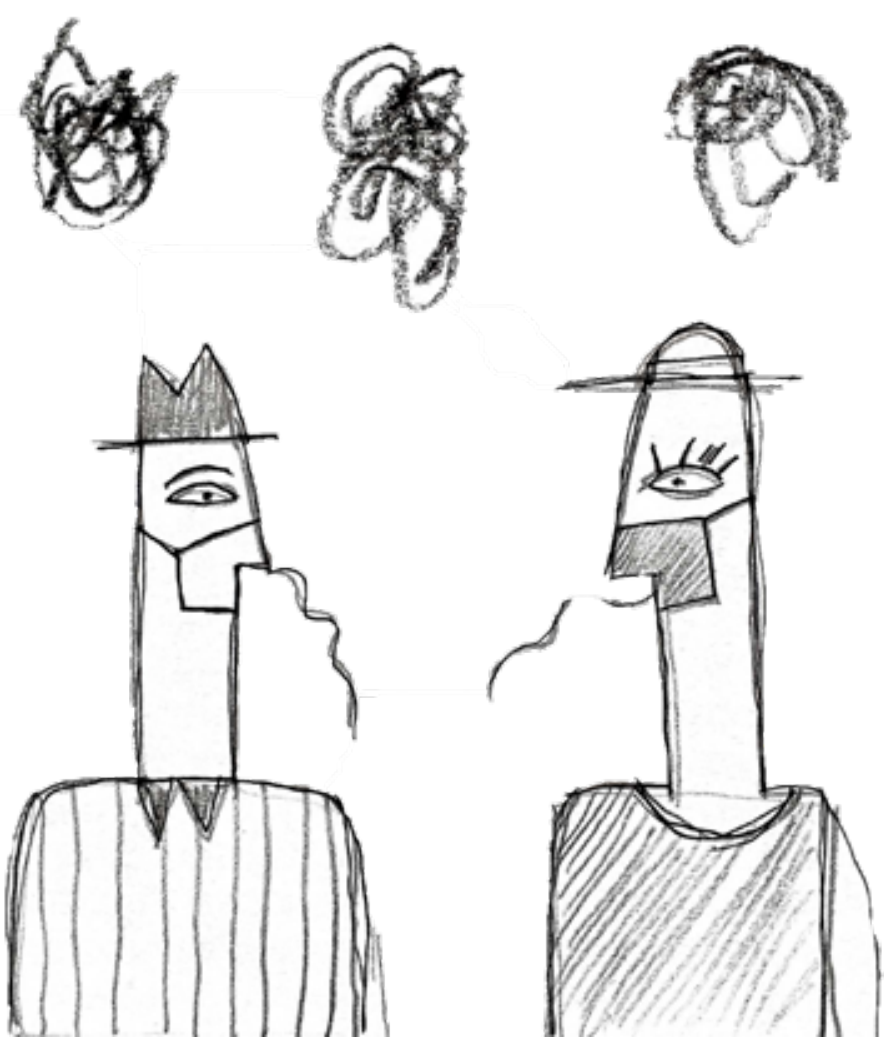

homogéneos entre sí, ni en el interior de las representaciones y prácticas; más aún, se distinguen por su heterogeneidad, contradicciones y ambivalencias (Menéndez Spina, 2009a).

5 Movimiento de temperancia y prohibicionismo en Estados Unidos y Yucatán a finales del siglo xix y principios del xx.

6 Periodo de la historia mexicana en la cual el país estuvo bajo el control militar de Porfirio Díaz, de 1876 a 1910. 


\section{Cuadro 1. Decretos emitidos por el Gobierno del Estado de Yucatán sobre la prohibición de la venta de bebidas alcohólicas (abril a noviembre de 2020), durante la contingencia sanitaria por covid-19}

\begin{tabular}{|c|c|c|c|}
\hline $\begin{array}{l}\text { Decreto } \\
{[\text { Fecha] }}\end{array}$ & Vigencia & Acción & Breve descripción / argumento \\
\hline $\begin{array}{l}\mathbf{2 0 8} / \mathbf{2 0 2 0} \\
{[9 \text { de abril] }}\end{array}$ & $\begin{array}{l}\text { Del } 10 \text { al } \\
30 \text { de abril }\end{array}$ & PROHIBICIÓN & $\begin{array}{l}\text { Prohibición en todo el estado en razón de la pandemia por SARS-Cov-2 (coviD-19) al } \\
\text { considerarse actividad no esencial, y para la prevención de la violencia doméstica }\end{array}$ \\
\hline $\begin{array}{l}\mathbf{2 1 3} / \mathbf{2 0 2 0} \\
{[29 \text { de abril] }}\end{array}$ & $\begin{array}{l}\text { Se extiende } \\
\text { al } 15 \text { de mayo }\end{array}$ & PROHIBICIÓN & $\begin{array}{l}\text { El } 21 \text { de abril el gobierno federal declaró fase } 3 \text { en todo el país; estatalmente los } \\
\text { contagios y fallecimientos por covid- } 19 \text { han aumentado }\end{array}$ \\
\hline $\begin{array}{l}217 / 2020 \\
{[14 \text { de mayo] }}\end{array}$ & $\begin{array}{l}\text { Se extiende } \\
\text { al } 31 \text { de mayo }\end{array}$ & PROHIBICIÓN & $\begin{array}{l}\text { El } 13 \text { de mayo Yucatán ocupaba la posición seis a nivel nacional de casos con- } \\
\text { firmados activos; las cifras estatales siguen en aumento }\end{array}$ \\
\hline $\begin{array}{l}\mathbf{2 2 6} / \mathbf{2 0 2 0} \\
{[30 \text { de mayo] }}\end{array}$ & $\begin{array}{l}\text { Del } 1 \text { al } \\
10 \text { de junio }\end{array}$ & $\begin{array}{l}\text { Reactivación } \\
\text { paulatina y } \\
\text { prohibición } \\
\text { parcial }\end{array}$ & $\begin{array}{l}\text { Limitar la movilidad y aglomeración de personas; se mantiene la prohibición en } \\
\text { establecimientos que expenden para consumo en el mismo lugar y se reactiva } \\
\text { para los que expenden para consumo en otro lugar, sujeto a disposiciones de } \\
\text { modalidad, horario habitual y cantidad* }\end{array}$ \\
\hline $\begin{array}{l}\mathbf{2 3 9} / \mathbf{2 0 2 0} \\
{[9 \text { de junio] }}\end{array}$ & $\begin{array}{l}\text { Del } 11 \text { al } \\
17 \text { de junio }\end{array}$ & $\begin{array}{l}\text { Reactivación } \\
\text { paulatina y } \\
\text { prohibición parcial }\end{array}$ & $\begin{array}{l}\text { Necesidad de reactivación paulatina para la recuperación económica, evitando } \\
\text { concentraciones; se mantienen las prohibiciones y disposiciones del decreto anterior }\end{array}$ \\
\hline $\begin{array}{l}\mathbf{2 4 0 / 2 0 2 0} \\
{[16 \text { de junio] }}\end{array}$ & $\begin{array}{l}\text { Del } 16 \text { al } \\
28 \text { de junio** }\end{array}$ & $\begin{array}{l}\text { Reactivación } \\
\text { paulatina y } \\
\text { prohibición parcial }\end{array}$ & $\begin{array}{l}\text { Ampliar el periodo de vigencia de acciones extraordinarias }(226 / 2020 \text {, ya modi- } \\
\text { ficado por el decreto } 239 / 2020) \text {, para la recuperación económica, sin relajar las } \\
\text { medidas sanitarias }\end{array}$ \\
\hline $\begin{array}{l}\mathbf{2 5 2} / \mathbf{2 0 2 0} \\
{[14 \text { de julio] }}\end{array}$ & $\begin{array}{l}\text { Del } 14 \text { de julio } \\
\text { al } 15 \text { de agosto }\end{array}$ & PROHIBICIÓN & Repunte del número de personas contagiadas de coviD-19 en Yucatán \\
\hline $\begin{array}{l}\mathbf{2 7 3} / \mathbf{2 0 2 0} \\
{[15 \text { de agosto] }}\end{array}$ & $\begin{array}{l}\text { Se extiende } \\
\text { (se aplica del } 14 \\
\text { al } 31 \text { de agosto) }\end{array}$ & PROHIBICIÓN & $\begin{array}{l}\text { Se han logrado ciertos avances en la contención de la covid-19 en Yucatán, pero } \\
\text { persisten condiciones que ameritan la continuación y el reforzamiento de estas } \\
\text { medidas }\end{array}$ \\
\hline $\begin{array}{l}\mathbf{2 7 5} / \mathbf{2 0 2 0} \\
{[29 \text { de agosto] }}\end{array}$ & 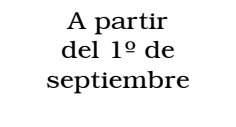 & $\begin{array}{l}\text { Reactivación } \\
\text { paulatina } \\
\text { y prohibición } \\
\text { parcial }\end{array}$ & $\begin{array}{l}\text { Se mantienen avances en la contención de la covid-19, pero persisten condicio- } \\
\text { nes que ameritan estas medidas, al mismo tiempo que la reactivación económica } \\
\text { es necesaria; mismas medidas que las establecidas el } 30 \text { de mayo pero se permite } \\
\text { la venta y consumo en restaurantes (medidas de seguridad y horario limitado) }\end{array}$ \\
\hline $\begin{array}{l}\mathbf{2 8 2} / \mathbf{2 0 2 0} \\
{[30 \text { de }} \\
\text { septiembre }]\end{array}$ & $\begin{array}{l}\text { A partir } \\
\text { del } 1 \text { o de } \\
\text { octubre }\end{array}$ & $\begin{array}{l}\text { Reactivación } \\
\text { paulatina }\end{array}$ & $\begin{array}{l}\text { Acciones extraordinarias para reactivar paulatinamente la venta y el consumo } \\
\text { de alcohol, como medida temporal de prevención sanitaria ante la emergencia } \\
\text { por covid-19 en Yucatán }\end{array}$ \\
\hline $\begin{array}{c}\mathbf{2 8 4} / \mathbf{2 0 2 0} \\
{[6 \text { de octubre] }}\end{array}$ & $\begin{array}{l}\text { A partir del } \\
\text { mismo día }\end{array}$ & PROHIBICIÓN & $\begin{array}{l}\text { Medida temporal de prevención sanitaria ante el paso del huracán Delta por el } \\
\text { estado de Yucatán y sus posibles afectaciones }\end{array}$ \\
\hline $\begin{array}{l}\mathbf{2 8 6} / \mathbf{2 0 2 0} \\
{[7 \text { de octubre] }}\end{array}$ & $\begin{array}{l}\text { Del } 8 \text { de } \\
\text { octubre al } 1^{\text {o }} \text { de } \\
\text { noviembre }\end{array}$ & Reactivación & $\begin{array}{l}\text { Acciones extraordinarias para reactivar paulatinamente la venta y consumo } \\
\text { de alcohol como medida temporal de prevención sanitaria por la afectación de } \\
\text { covid-19 en Yucatán }\end{array}$ \\
\hline $\begin{array}{l}\mathbf{2 8 8} / \mathbf{2 0 2 0} \\
{[26 \text { de octubre] }}\end{array}$ & $\begin{array}{l}\text { A partir del } \\
\text { mismo día }\end{array}$ & PROHIBICIÓN & $\begin{array}{l}\text { Medida temporal de prevención sanitaria ante el paso del huracán Zeta por el } \\
\text { estado de Yucatán y sus posibles afectaciones }\end{array}$ \\
\hline $\begin{array}{l}\mathbf{2 8 9} / \mathbf{2 0 2 0} \\
{[27 \text { de octubre] }}\end{array}$ & $\begin{array}{l}\text { A partir del } \\
\text { mismo día }\end{array}$ & Reactivación & $\begin{array}{l}\text { Acciones extraordinarias para reactivar paulatinamente la venta y consumo de } \\
\text { alcohol como medida temporal de prevención sanitaria ante la afectación por } \\
\text { COVID-19 en Yucatán. }\end{array}$ \\
\hline $\begin{array}{c}\mathbf{2 9 0} / \mathbf{2 0 2 0} \\
{\left[1^{\circ} \text { de noviembre] }\right.}\end{array}$ & $\begin{array}{l}\text { Del } 2 \text { al } 30 \\
\text { de noviembre }\end{array}$ & Reactivación & $\begin{array}{l}\text { Modificación del decreto } 289 / 2020 \text {, mismos argumentos; venta y consumo en } \\
\text { establecimientos y lugares autorizados }\end{array}$ \\
\hline \multicolumn{4}{|c|}{$\begin{array}{l}\text { * Venta máxima por persona de hasta: } 24 \text { latas, “cuartitas" o "medias", nueve "caguamas", siete "misiles" y hasta una botella de } 1250 \text { milili- } \\
\text { tros de vinos o licores. Sobre la Entrega y Recepción (Art. 3) el decreto } 226 / 2020 \text { señala que será únicamente a cargo de diligencieros (del } \\
\text { expendio y/o de plataformas, debidamente identificados), sólo a mayores de edad con identificación, y queda "prohibido entregar bebidas } \\
\text { alcohólicas a personas en visible estado de ebriedad". } \\
\text { " A partir del } 29 \text { de junio se "normalizó" la venta de bebidas alcohólicas en establecimientos que expendan para su consumo en otro lugar, } \\
\text { no así en lugares para consumo en el mismo lugar. }\end{array}$} \\
\hline
\end{tabular}

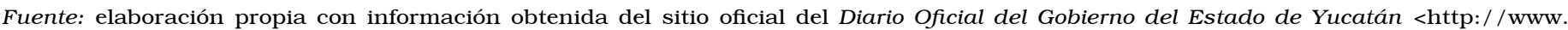
yucatan.gob.mx/gobierno/diario_oficial.php>. 
Los llama "gobiernos" porque reflejan la complejidad y diversidad de estas políticas, pero también por su alusión al supuesto dominio que se creía -y se cree-debía existir sobre uno mismo, sobre los propios deseos, sobre el cuerpo y sobre la manera de actuar (Márquez Osuna, 2014). Retomaré esta idea en el siguiente apartado cuando se discuta la "dependencia" al consumo colectivo de alcohol.

La otra racionalidad gubernamental que destaca en los decretos oficiales es el reconocimiento exclusivo de los perjuicios de este consumo, planteamientos que, sin negarse, ignoran u "olvidan" (Menéndez Spina, 2009b) que, en los hechos, el consumo sociocultural de alcohol conlleva no sólo a consecuencias negativas, sino también a consecuencias positivas para los sujetos y conjuntos sociales.

Esto apunta a una falta de comprensión científico-social en las acciones gubernamentales que compromete su efectividad. Así, mientras por un lado se le atribuye al alcohol una serie de "vicios" de los que no es responsable directo, como en el caso de la relación alcohol/violencias, ${ }^{7}$ por otro se simplifica la comprensión de su aspecto negativo, como en el caso de las intoxicaciones agudas por alcohol y de los accidentes vehiculares que, pese a sus elementos socioculturales, son entendidos y atendidos predominantemente desde una perspectiva criminal y/o patológica (Ronzani y Touzé, 2020).

Pero además, si lo negativo de este consumo fuese tan obvio, y su prohibición tan consensuada, habría un apoyo general a estas medidas. En cambio, resaltó un entramado de opiniones encontradas de las cuales retomo una muestra. ${ }^{8}$ A favor de la prohibición se podían leer comentarios como: "perfecto [...] ese dinero que van a malgastar en cerveza bien lo pueden invertir en despensa [refiriéndose a las personas bebedoras]", "sufran adictos, el único líquido esencial es el agua" y "Me encanta ver como se enojan los alcohólicos [...] a ver si así cumplen en mantener bien a sus familias".

Por el contrario, entre las opiniones críticas de la ley seca se leían: "malísima idea, ya que se desatará la abstinencia en los hogares donde predomina violencia intrafamiliar", "Ley seca de coca o refrescos embotellados que hace más mal que una cerveza”, "para mí sí es esencial" y "que les regresen su trabajo a los de las cerveceras, no piensan en sus familias, ellos también tienen que comer y llevar dinero a sus casas".

El apoyo o rechazo expresado en estas opiniones sobre la "ley seca" no descansa sólo en saberes biomédicos, sino en saberes que entrelazan lo moral con lo ideológico y, a su vez, con lo biomédico, y de lo que resultan aparentes contradicciones que confirman la necesidad de esta restricción, pero que al mismo tiempo reconocen y hasta defienden los beneficios de este consumo, como lo corrobora la venta clandestina de bebidas alcohólicas (Rico, 2020; Diario de Yucatán, 2020).

Todavía más, un par de días antes de que el primer decreto prohibicionista entrase en vigor, se rumoró que familiares, amistades y funcionarios allegados al gobierno estatal fueron prevenidos de la inminente ley seca, para que pudieran abastecerse de bebidas, alertamientos que en más de una ocasión derivaron en compras "de pánico" de alcohol (La verdad, 2020; Barquet Loeza, 2020). Este rumor lo confirmé después, de manera extraoficial, con personas cercanas al gobierno, que corroboraron la advertencia. Ello muestra que la "contradicción" no es exclusiva de un solo sector social e incluso que, quienes enuncian pública y oficialmente su predominante carácter negativo, en los hechos también reconocen lo positivo que hay en él.

Pero esta aparente contradicción no es otra cosa que una postura ambivalente que forma parte de las representaciones $\mathrm{y}$ prácticas sociales yucatecas -o por lo menos de una parte de la población- sobre el consumo de alcohol y de sus consecuencias. O, dicho de otra forma: en Yucatán, el alcohol y su consumo se reconocen y viven como algo "bueno" y como algo "malo" simultáneamente (Moreno Cabrera, 2020a).

Uno de los efectos negativos e inesperados de esta medida fue el fallecimiento de 16 personas en Yucatán (Yucatán Ahora, 2020), en su mayoría hombres, a causa de una severa intoxicación por consumir alcohol adulterado y/o por mezclar sus bebidas con gel antibacterial, ante la imposibilidad de poder comprar sus bebidas de forma legal. En toda la república mexicana, y para finales de mayo, la cifra llegaba a casi 160 fallecimientos por estos mismos motivos (Miranda, 2020). Este dato no es menor si consideramos

\footnotetext{
7 En otro trabajo analizo y discuto localmente la función del consumo de alcohol como chivo expiatorio de las violencias de género, tanto en el plano estructural como cotidiano (Moreno Cabrera, 2020b). Pero valga decir, además, que las prohibiciones alcohólicas no contribuyeron a reducir la violencia contra las mujeres en los hogares durante el primer año de la pandemia; es más, diferentes organizaciones e instancias de atención a mujeres, sus hijos e hijas, en situación de violencia, registraron un aumento de 48 por ciento de atenciones de marzo a noviembre de 2020, en comparación con el año anterior (Red Nacional de Refugios, véase: https: / / rednacionalderefugios.org.mx/).

8 Comentarios en la red social Facebook del Diario de Yucatán, de la publicación del 9 de abril del 2020.
} 
que en 2018, en todo Yucatán, 19 personas fallecieron por síndrome de dependencia alcohólica, ${ }^{9}$ todos hombres de 35 a 64 años de edad, sin reportarse muertes femeninas por esta razón, al menos no entre las primeras veinte causas de mortalidad de ese año. Es decir que, en tan sólo unas semanas, las muertes por intoxicación aguda con alcohol adulterado o gel antibacterial casi igualan el número de muertes por dependencia alcohólica de todo 2018.

Una explicación parcial sería responsabilizar de estas muertes a los propios alcohólicos, por no "saber" controlar sus impulsos, por "no saber parar". Pero, por obvia o tentadora que resulte, no es otra cosa que una explicación parcial, pues "olvida" que no todas las personas que beben alcohol, ya sea con frecuencia o incluso diariamente, son "adictas" o presentan dependencia alcohólica en términos clínico-biomédicos. Entonces, si no todas las personas que beben hasta intoxicarse son “alcohólicas”, ¿por qué no detienen su ingesta antes de sufrir estos daños o incluso la muerte?

Una posible respuesta la encontramos "paradójicamente" en lo que considero un efecto positivo pero no intencional de la ley seca: la disminución significativa y sostenida del número de intoxicaciones agudas por alcohol en Yucatán durante 2020 y en comparación con 2019 y con años anteriores (véanse gráficas la y 1 b y 2 a y 2 b); esto es, la reducción del número de personas que tras beber altas cantidades de alcohol, en dosis únicas o múltiples, y en poco tiempo, tuvieron consecuencias negativas en su salud física (Rodríguez González, 2015). ${ }^{10}$

Mi hipótesis sobre la relación prohibición/reducción de intoxicaciones la respondo con la información etnográfica presentada en el siguiente apartado. Pero por ahora quiero subrayar que la reducción descansaría en una de las principales características socioculturales del consumo de alcohol en Yucatán: la mayoría de la población bebedora prefiere consumir alcohol de forma colectiva y no en solitario.

Esto no excluye que tendría que explorarse si el temor a contraer COVID-19 disuadió a la población y personas que sufrieron de intoxicaciones agudas por alcohol durante el confinamiento de acudir a un servicio de salud. Pese a lo anterior, pienso que la reducción significativa de casos de intoxicación aguda por alcohol en Yucatán no podría explicarse únicamente desde la sensación o percepción de riesgo en la población, como se observa en los gráficos.

En este sentido, las cifras llaman la atención. Si comparamos el incremento del número de casos semanales acumulados de estas intoxicaciones, vemos que la tendencia es relativamente similar entre ambos años y hasta la semana epidemiológica número 15, sumando para ese entonces 2339 personas intoxicadas en 2019 y 1978 en 2020. Pero justo a partir de la semana 15 de 2020, y como resultado de la implementación de las prohibiciones y restricciones alcohólicas, se observa un importante decrecimiento y estancamiento en el número de casos acumulados de hombres y mujeres yucatecas.

No obstante, y retomando la intención de los gobiernos de alcohol prohibicionistas, ya discutida, no podríamos asegurar que estas restricciones tuvieron un impacto determinante ni en la reducción del consumo de alcohol ni en el número de contagios por Covid-19, ya que, como muestran las gráficas $2 a$ y $2 b$, no parece existir una correlación significativa entre las prohibiciones y el número de contagios semanales detectados.

En síntesis, estos cuestionamientos no buscan desacreditar la importancia de una respuesta oficial ante emergencias sanitarias como la pandemia por COVID-19, pero la implementación de cualquier medida, en especial de tipo gubernamental y referida a procesos de $\mathrm{s} / \mathrm{e} / \mathrm{a}-\mathrm{p}$ tan complejos como el consumo de alcohol y sus consecuencias, demandan una lectura más profunda y comprometida de las características del contexto sociocultural que lo configuran, y siempre en diálogo con los saberes biomédicos.

\section{Entre lo "malo" y lo "bueno", entre lo ideal y lo real}

En el presente apartado busco ampliar la discusión sobre las representaciones sociales subyacentes a las medidas prohibicionistas enunciadas por los gobiernos, y debatidas socialmente, a partir de la información etnográfica obtenida durante mi trabajo de campo doctoral (Moreno Cabrera, 2019). En este sentido, lo

9 Instituto Nacional de Estadística y Geografía (INEGI) <https://www.inegi.org.mx/programas/mortalidad/default.html\# Tabulados>.

${ }^{10}$ Hay que recordar que estas cifras dan cuenta de los casos que llegan y son atendidos en cualquiera de los servicios de salud de la entidad, dejando fuera a todas aquellas personas que no pueden o deciden no acudir a los servicios sanitarios. Rodríguez González (2015) puntualiza que existen cuatro grados de intoxicación, pasando de la excitación (embriaguez) y la confusión, hasta el estupor, el coma e incluso la muerte. Véase también la definición de la Organización Mundial de la Salud al respecto: <https://icd.who.int/browse 11/1-m/es\#/http\%3a\%2f\%2fid.who.int\%2ficd\%2 fentity\%2f1339202943>. 


\section{Gráfica 1a. Número de casos de intoxicación aguda por alcohol en hombres y mujeres de Yucatán, 2019}

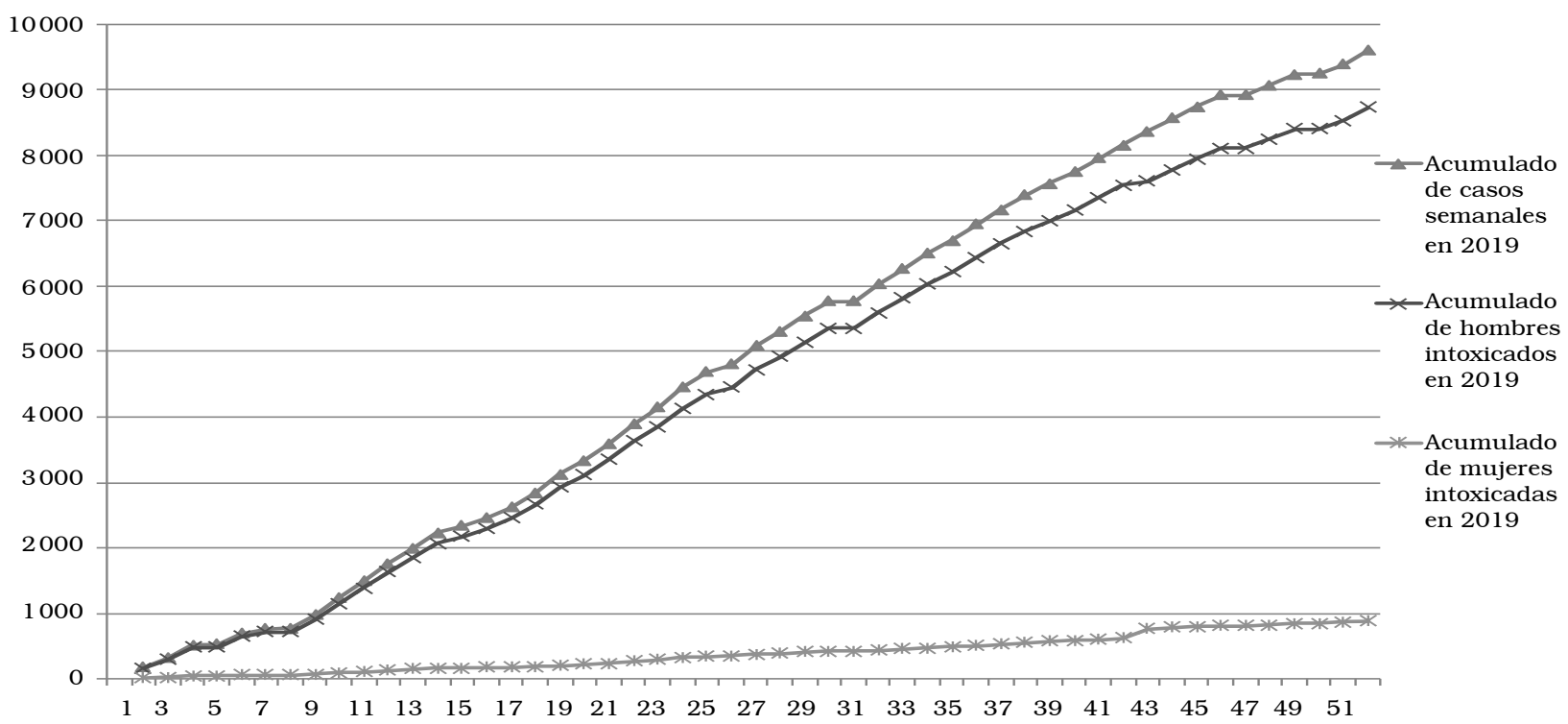

Fuente: elaboración propia con base en datos de Boletín Epidemiológico del Sistema Nacional de Vigilancia Epidemiológica Sistema Único de Información, Secretaría de Salud de México (Boletines 2 a 52 del 2019) <https://www.gob.mx/salud/ documentos / boletinepidemiologico-sistema-nacional-de-vigilancia-epidemiologica-sistema-unico-de-informacion-231750>.

\section{Gráfica 1b. Número de casos de intoxicación aguda por alcohol en hombres y mujeres de Yucatán, 2020}

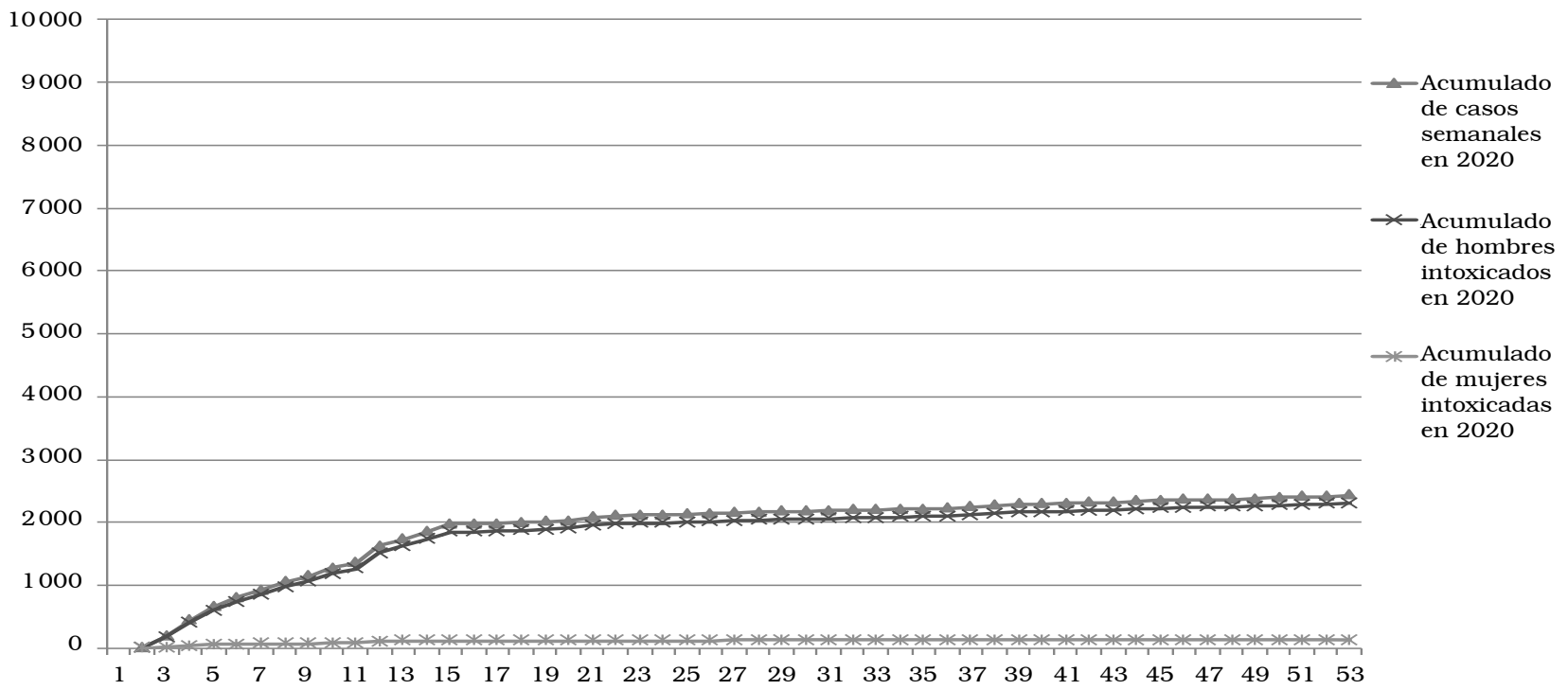

Fuente: elaboración propia con base en datos de Boletín Epidemiológico del Sistema Nacional de Vigilancia Epidemiológica. Sistema Único de Información, Secretaría de Salud de México (Boletines 2 a 53 de 2020) <https://www.gob.mx/salud/ documentos / boletinepidemiologico-sistema-nacional-de-vigilancia-epidemiologica-sistema-unico-de-informacion-231750 > 
Gráfica 2a. Interacción de las prohibiciones alcohólicas (ley seca) yucatecas de 2020 con el número de casos de intoxicación aguda por alcohol en el mismo año

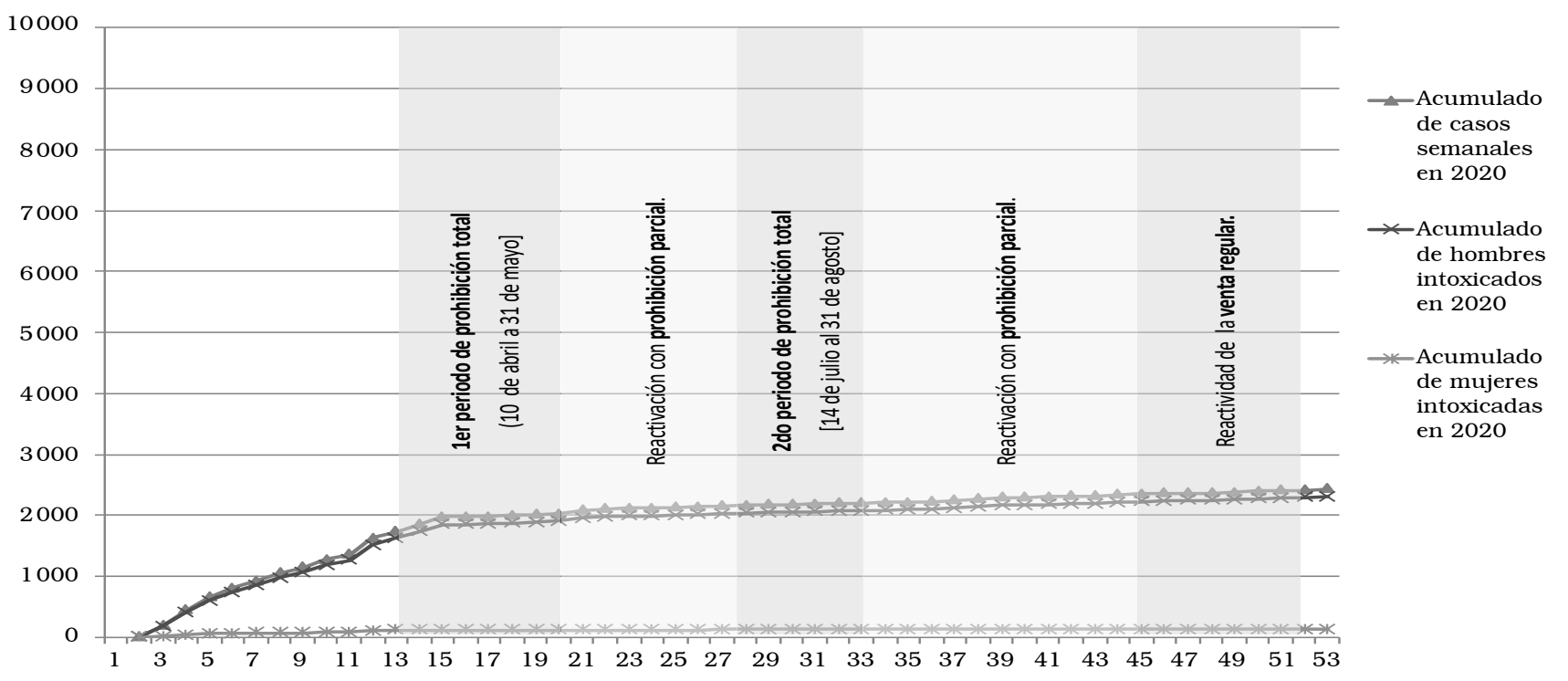

Fuente: elaboración propia con base en datos de Boletín Epidemiológico del Sistema Nacional de Vigilancia Epidemiológica. Sistema Único de Información, Secretaría de Salud de México (Boletines 2 a 52 del 2019) <https://www.gob.mx/salud/ documentos / boletinepidemiologico-sistema-nacional-de-vigilancia-epidemiologica-sistema-unico-de-informacion-231750> y Boletín Epidemiológico/DGE/DIE/Covid-19. Casos confirmados por laboratorio.

\section{Gráfica 2b. Interacción de las prohibiciones alcohólicas (ley seca) yucatecas de 2020 con el número de casos de covid-19 detectados el mismo año}

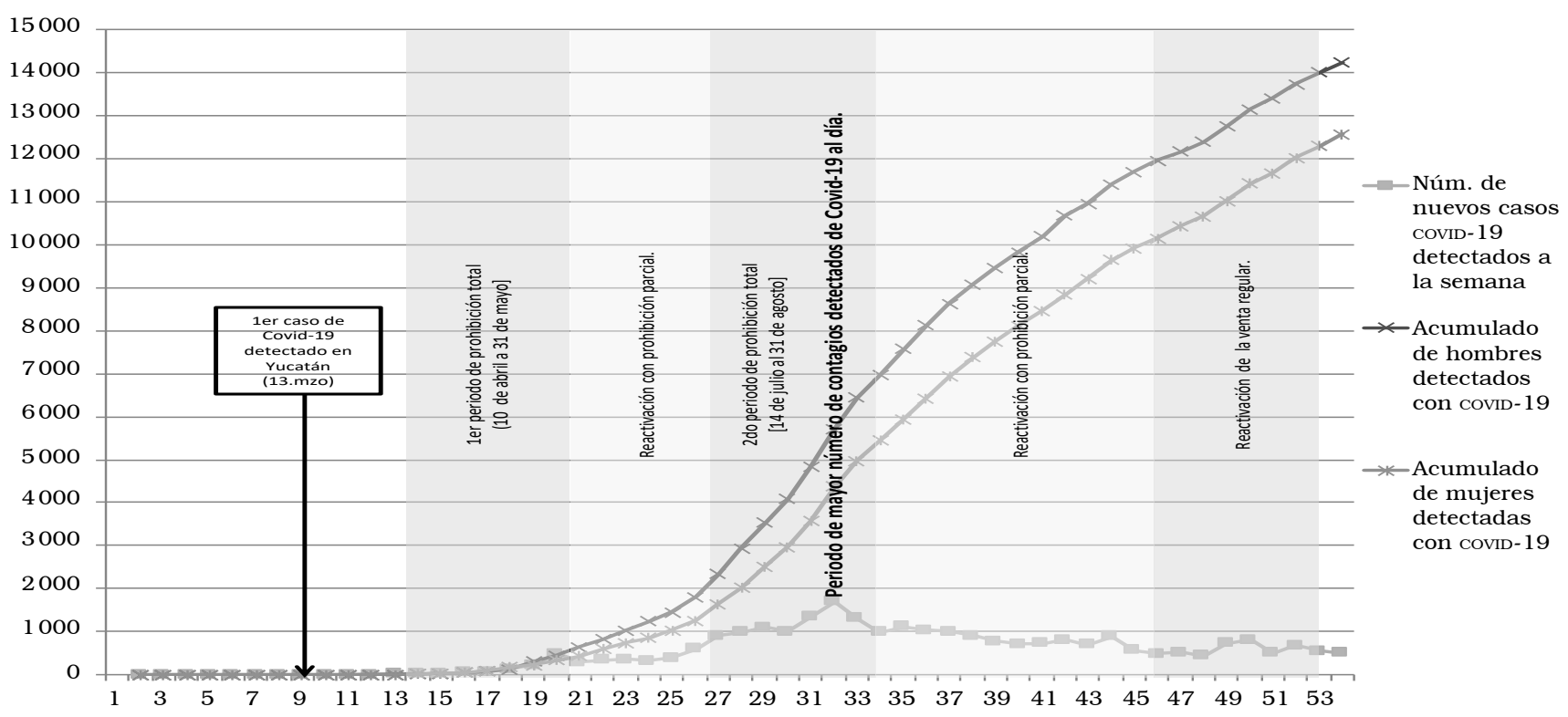

Fuente: elaboración propia con base en datos de Boletín Epidemiológico del Sistema Nacional de Vigilancia Epidemiológica. Sistema Único de Información, Secretaría de Salud de México (Boletines 2 a 53 de 2020) <https://www.gob.mx/salud/ documentos / boletinepidemiologico-sistema-nacional-de-vigilancia-epidemiologica-sistema-unico-de-informacion-231750> y Boletín Epidemiológico/DGE/DIE/Covid-19. Casos confirmados por laboratorio. 
más destacado de la etnografía desarrollada en Cuzamá fue la preponderancia de la representación negativa sobre el consumo de alcohol.

Expresiones como “¿qué tiene de bueno el alcohol”, "No hay nada bueno en el alcohol" o "estamos en primer lugar de alcoholismo en todo Yucatán", imperaban en los discursos de la población, y sin duda hallaban su correlato en las lesiones y muertes por accidentes vehiculares, en la vinculación consumo/suicidios (no como causa directa, pero sí reconocida como un factor agravante), en la enfermedad o muerte por cirrosis hepática alcohólica, o en las violencias alcoholizadas (discusiones, pleitos, golpes); pero estas consecuencias no eran las predominantes.

Conforme la confianza aumentaba, los actores ${ }^{11}$ (hombres y mujeres) admitían, y me mostraban en la convivencia, las consecuencias positivas que este consumo tenía en sus vidas y en su salud física, emocional y relacional, todo lo cual procuraban y disfrutaban cuando bebían de forma colectiva y sobre todo con su grupo de pares, la "banda". De esta forma, los saberes sobre las consecuencias del consumo de alcohol en Cuzamá y su relación con el proceso de s/e/a-p resaltaban principalmente su carácter ambivalente (Moreno Cabrera, 2020a).

Ambos sexos identificaban y reconocían afectaciones en su salud, derivadas de episodios prolongados y de alto consumo, aunque con diferencias de género; pero, al mismo tiempo, admitían que en la mayoría de estas ocasiones experimentaban beneficios en su salud física y emocional ("desestrés”, diversión) y relacional (convivencia, expresión de afectos e interacciones no violentas), además de las ganancias económicas que la venta de alcohol representaba para el gobierno municipal y para casi un tercio de la población (empleados en restaurantes y bares de la ciudad).

La ambivalencia no implica que no hubiera un reconocimiento de lo negativo y su distinción con lo positivo, es más, las categorías locales "saber tomar" y "no saber tomar" confirmaban la existencia de un consenso sociocultural sobre el consumo permitido y el sancionado (Moreno Cabrera, 2020a). La ambivalencia era también distinta a aquellas ocasiones en las cuales lo que ocurría era un "acomodo intencional" según la situación social (Gilbert, 1985), como cuando se juzgaba más el consumo de las mujeres en comparación con el de los hombres, pese a que el primero fuera menor en cantidad, frecuencia o impactos -0 incluso fuera igual.
Es decir, la ambivalencia devela algo más profundo: un sistema sociocultural estructural y estructurante (dialéctico) del que no siempre se tiene conciencia, pero que mantiene y reproduce determinados procesos como el de la vinculación alcohol/salud-enfermedad, esto es, donde lo "bueno" y lo "malo" coexisten, y donde lo que se dice no siempre se corresponde con lo que se hace. En otras palabras, se reconoce que ciertas formas de beber pueden afectar la vida, salud y relaciones sociales y al mismo tiempo derivar en beneficios también para la salud y las relaciones sociales.

Varios de los hombres con quienes trabajé -a diferencia de las mujeres-venían de trayectorias de consumo de alcohol de hasta 20 años, y con una frecuencia promedio de consumo quincenal o semanal. Muchos empezaron a beber a los 14 o 15 años con sus amigos y, aunque no lo hacían con igual intensidad con la que beben en la actualidad, la ritualidad de esta práctica y el tiempo han ido aumentando su tolerancia, como lo explica Kike (31 años): "a los 15 [años] estás en tu apogeo, tu hígado está agarrando callo, no te afecta; pero llegando a los 25 años o más, ya tronaste... decaes, ya no es lo mismo".

Miguel (35 años), uno de los mejores amigos de Kike y con una trayectoria de 20 años de consumo semanal promedio, me platicó que un par de años atrás requirió de hacerse unos análisis de sangre por motivos de trabajo. Sus resultados lo impactaron o al menos en cierta medida: "todo salió mal", me compartió, y aunque el médico lo exhortó a reducir su consumo de alcohol la sugerencia fue desestimada por Miguel, "a mí me gusta mi vida como es... y yo así me voy a morir”, sentenció.

Liz (31 años) me compartió sobre las dos ocasiones cuando ella, sus hijos y esposo habían estado cerca de accidentarse en una camioneta a su regreso de una fiesta popular en otro poblado, a 40 minutos de Cuzamá. Un amigo de su esposo y éste venían al frente, uno manejando y el otro como copiloto, ambos alcoholizados y bebiendo mientras manejaban. Pese al reconocido riesgo y temor de que esto pueda volver a ocurrir, Liz y su familia repiten esa actividad por lo menos una vez al mes, "nos gusta mucho ir a las fiestas y corridas de toros".

Para Beatriz Cortés (1988), un análisis complejo y relacional de la alcoholización debe dar cuenta de la coexistencia de lo positivo y lo negativo, pues es justo esta multifunción la que asegura su continuidad pese a sus contradicciones. La autora enfatiza que ambos

\footnotetext{
${ }^{11}$ Los actores sociales son sujetos que, aunque ejercen una identidad y subjetividad particular, no se representan sólo como individuos, sino como sujetos inmersos en relaciones sociales; pero, además, no sólo informan lo que piensan sobre una problemática, sino que también la actúan, la viven (Menéndez Spina, 2009a; Moreno Cabrera, 2019).
} 
aspectos (lo positivo y lo negativo) son influidos por el contexto y cambian históricamente según los procesos económico-políticos e ideológicos dominantes, es decir, que son configurados de forma subjetiva y colectiva en y desde la estructura social.

Desde una mirada transcultural, Heath (2012) plantea que el consumo de alcohol opera como una "ventana" hacia la cultura o, retomando a Duster, "el alcohol es a la ciencia social lo que la tinta al microscopio... la tinta muestra ciertos tipos de características fundamentales de las estructuras de la célula, [...] probablemente podamos usar el alcohol de la misma manera, para penetrar en la estructura de la vida social" (cit. en Heath, 2012: 106). ${ }^{12}$ Es desde esta misma mirada que he analizado en otro trabajo las desigualdades de género y los privilegios masculinos cotidianos que subyacen a los saberes sociales sobre el consumo de alcohol en Yucatán (Moreno Cabrera, en prensa).

El estudio sociocultural de los procesos de s/e/a-p nos permite problematizar rituales cotidianos como el consumo de alcohol y resaltar los procesos estructurales que le subyacen, el "intricado y entretejido sistema de patrones que llamamos cultura" (Heath, 2012: 106). Linton (1945) nombraba pautas culturales a aquellos patrones que los sujetos aprendemos (socialización) para orientar nuestras acciones en el interior del grupo social, garantizando así la reproducción de la sociedad misma; Linton destaca entre éstas las pautas ideales y las pautas reales.

Las pautas culturales ideales son las abstracciones creadas y consensuadas por la propia sociedad sobre lo que debe ser el comportamiento de los sujetos en determinadas situaciones, mientras que las reales se constituyen por las actitudes y acciones manifiestas, lo que de hecho sucede y que, en última instancia, reproduce la vida social. Pero ese autor señala que lo real no siempre se corresponde con lo ideal, ya sea porque este último resulta imposible o porque se trata de un valor que, pese a su aceptación y reconocimiento, se ignora, no se concreta o se infringe más de lo que se pregona (Linton, 1945).

En este orden de ideas, Heath (2012) y Menéndez Spina (2020) han cuestionado las mediciones cuantitativas -particularmente las encuestas- que aseguran conocer cuánto alcohol bebe una población determinada, olvidando que lo que sus instrumentos pueden llegar a registrar en todo caso son las "normas del" comportamiento alcoholizado o lo que debe decirse (lo ideal), mientras que los científicos sociales tienen la posibilidad de acceder a las "normas sobre" el comportamiento alcoholizado o lo que de hecho ocurre (lo real).

En síntesis, las representaciones y prácticas sobre el consumo de alcohol en Cuzamá confirman la existencia de un sistema sociocultural que reconoce y vive esta ingesta de forma positiva y negativa, y es esta ambivalencia la que sostiene y reproduce esta forma de alcoholización, es decir, un consumo "idealizado", donde el alcohol debe enunciarse formalmente como algo "malo", pero que plantea una línea divisoria-moral e ideológica- que separa a aquellos que "saben tomar" de quienes "no saben tomar". Sin embargo, y en la práctica, el consumo colectivo en Cuzamá -y quizá en todo Yucatán- se desarrolla entre "lo bueno y lo malo", entre "lo ideal y lo real", en una ambigüedad que, aunque no se admita, caracteriza a éste y tal vez a otros procesos de la cultura yucateca. En otro trabajo describo con mayor detalle esta ambivalencia (véase Moreno Cabrera, 2020a).

\section{¿Dependencia individual al alcohol o al consumo colectivo de alcohol?}

El consumo de alcohol en Cuzamá apareció siempre como una acción social, no sólo porque se "aprendía" y configuraba dentro de relaciones sociales (familiares, de pareja, amicales y comunitarias), sino porque se vivía como una práctica predominantemente colectiva y no solitaria. Los hombres y mujeres con quienes trabajé -y la población en general-, demostraban una y otra vez que no les gustaba beber solos ni solas: "iay no! tomar sola no me gusta” (Olga, 34 años); "no sabe igual" (Ana, 27 años), "es triste" (Leo, 26 años; Memo, 35 años).

Uno de los actores secundarios (32 años) me explicaba en una ocasión que, a veces, con tal de no gastar mucho dinero con sus amigos y evitar así conflictos con su esposa, se compraba un "six"13 de cervezas para ver el futbol en casa: "preparo todo: enfrío mis cervezas, hago mis botanas, me acomodo... abro mi cerveza... la empiezo, pero no me la acabo... es que solo no es lo mismo". En contraste y en compañía de su "banda", podía beber hasta seis o más litros de cerveza en una sola ocasión.

Esta preferencia por el consumo colectivo la observé en todos los hombres y mujeres bebedoras de alcohol con quienes conviví y a quienes entrevisté. "No es el sabor, sino el ambiente", dijo Memo (35 años) para explicar que no era la sustancia per se,

\footnotetext{
12 Traducción propia.

${ }^{13}$ Paquete de seis latas de cerveza de $355 \mathrm{ml}$ cada una.
} 
sino el contexto relacional que se creaba de manera colectiva, y los efectos y afectos que en éste sucedían. Se trataba de un ambiente de confianza y diversión con "la banda", ${ }^{14}$ que si bien no era exclusivo de las ocasiones de consumo, en éstas solía expresarse más; y aunque beber en colectivo también sucediera en otras relaciones como las familiares y de pareja, era principalmente en las relaciones amicales y comunitarias donde se procuraba y disfrutaba más.

"Vamos a tomar dos" era el llamado social al consumo colectivo, y éste no aludía a una cantidad exacta sino a su acción y obligación social. Esto es importante pues la obligatoriedad operaba lo mismo como un beneficio que como un perjuicio para la salud integral (física, emocional y relacional) de la mayoría de las personas. Su frecuencia dependía del tiempo libre de cada persona y grupo, y descansaba en la oposición "borracho"/"tomador" y responsabilidad/ irresponsabilidad.

Hombres y mujeres aseguran que los "borrachos" (los consumidores "enfermos", "adictos" o "alcohólicos”) eran quienes bebían todos los días y que, en consecuencia, incumplían con sus responsabilidades parentales, conyugales y/o comunitarias; en cambio, el hombre o la mujer "tomadora" sólo consumía ocasionalmente, una o dos veces por semana, debido a sus "responsabilidades". Y si bien esto podía ser cierto en algunos contextos de consumo, cuando se bebía con el grupo de pares muchas y muchos “tomadores” bebían como "borrachos".

"Vamos a tomar dos" podía significar, según el ambiente y la relación social, dos latas, tres "caguamas" (litro de cerveza), cuatro "misiles" (botellas de cerveza de 1.2 litros) o dos y más "cartones" de cerveza (cajas de 12 o 24 botellas de cerveza según presentación). En una comida de fin de semana con la familia, algunas personas compraban dos o tres caguamas para "tomar tranquilos y no pasarse" [embriagarse], pero, con la banda, había la expectativa y hasta una planeación anticipada de beber por varias horas y en altas cantidades.

A mayor consumo, mayor ambiente y a mayor ambiente mayor consumo. Los grupos de hombres, a diferencia de los de mujeres, llegaban a beber desde seis hasta 12 horas seguidas, intercalando su ingesta de alcohol con botanas y comida, y algunos de ellos de cinco hasta diez litros de cerveza por ocasión -en el caso de algunas mujeres el consumo podía llegar a cinco litros de cerveza por ocasión (Moreno Cabrera, 2019 y en prensa). Y es que el disfrute implicaba la corresponsabilidad de dar "la tanda", es decir, de de- volver el gesto e invitar al grupo con una ronda de "latas" o caguamas, lo que en palabras de Heath (2012) se refiere a la "etiqueta" o "parafernalia" del consumo social de alcohol, rituales sin los cuales sería menos placentero o menos apropiado socialmente.

Ser invitado garantizaba que cuando no se tenía dinero el grupo amortiguaría el propio consumo, pero, de la misma forma, cuando se tenía dinero se debía responder a la acción con igual generosidad con la que se recibió; no hacerlo, genera molestias y tensiones. Este aspecto juega un papel esencial en la ecuación del consumo prolongado y excesivo, pues mientras más personas y mayor ambiente, mayor podía ser la obligación y mayor la cantidad que se bebiera y, con ésta, mayor la posibilidad de experimentar alguna de sus consecuencias negativas inmediatas (accidentes, discusiones, pleitos) o, a largo plazo, desgaste y deterioro del cuerpo, comorbilidades o cirrosis alcohólica.

Tomando esto en cuenta, propongo una discusión sobre los alcances y límites de la noción de dependencia alcohólica. Desde la biomedicina (medicina, psiquiatría, psicología), esta dependencia se ha planteado en términos de una adicción patológica individual con variadas consecuencias para la salud y la vida social de los individuos afectados. Sin negar lo anterior, el primer límite de esta noción es que poco refleja cómo bebe la mayoría de la población (Menéndez Spina, 2020; Heath, 2012).
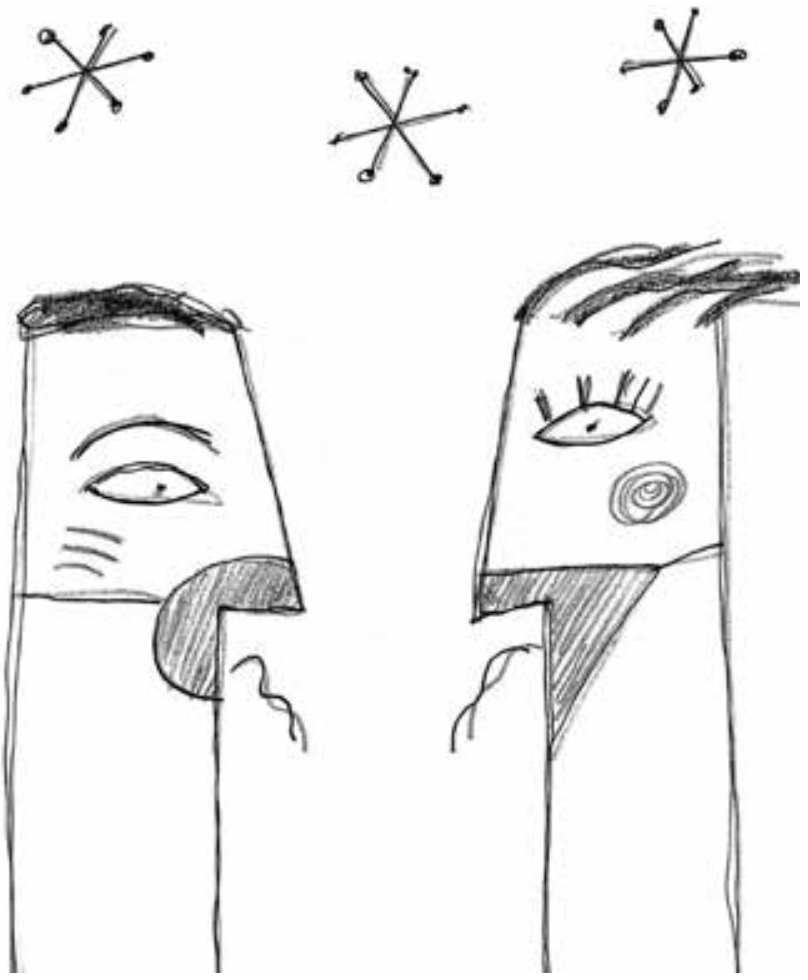

${ }^{14}$ Para una mayor descripción véase Moreno (2019). 
La última Encuesta Estatal de Adicciones de Yucatán (2014-2015) indica que 46 por ciento de la población encuestada reconoció beber alcohol en el último año, pero sólo 8.1 por ciento reportó dependencia. De forma similar, en la última Encuesta Nacional de Consumo de Drogas, Alcohol y Tabaco (Encodat) (Villatoro-Velázquez et al., 2017), 33.6 por ciento de la población encuestada de 12 a 65 años admitió consumo excesivo en el último año (45.5 por ciento hombres, 22.6 por ciento mujeres) y 19.8 por ciento en el último mes (29.9 por ciento hombres, 10.3 por ciento mujeres), mientras que sólo 2.2 por ciento presentó indicadores de dependencia (3.9 por ciento hombres, 0.6 por ciento mujeres).

Es decir, nuestra atención y problematización sobre las consecuencias (positivas y negativas) del consumo de alcohol debiera ponerse en la mayoría de los conjuntos sociales que se reconocen como bebedores habituales, y no de forma exclusiva en el reducido porcentaje de personas que presentan una adicción farmacológica (Menéndez Spina, 2020; Moreno Cabrera, 2019). Heath (2012) advierte que el consumo excesivo debe definirse a partir de cada contexto cultural, pues así como hay sociedades donde el consumo rara vez llega a la ebriedad hay otras donde las personas no se imaginan bebiendo sin embriagarse, patrones que desde la salud pública han clasificado a las culturas en "húmedas" y "secas”, siendo México y Cuzamá el caso de estas últimas (Medina-Mora, Berenzon y Natera Rey, 1999).

Que el consumo excesivo y la ebriedad se definan también desde su dimensión sociocultural no niega su afectación en la salud pública. Así, y a partir de los 45 años de edad, la cirrosis hepática alcohólica se ubica entre las primeras cinco causas de mortalidad en hombres yucatecos, y, además, las muertes indirectas a este consumo, como los suicidios y los accidentes motorizados, se colocan entre las primeras causas de mortalidad entre los hombres yucatecos desde temprana edad ${ }^{15}$ (Moreno Cabrera, 2019); y no se diga de los casos de intoxicación aguda por alcohol que han posicionado al estado en primer lugar nacional desde 2005, en el caso de los hombres, y desde 2013 en el caso de las mujeres, registrándose un total de 9779 casos en 2019 (8881 hombres, 898 mujeres).

La evidencia cuantitativa y cualitativa confirman que aunque la población yucateca -y mexicana- en general no reporta altos índices de dependencia alcohólica en tanto patrón de consumo predominante, lo cierto es que prevalece un patrón ocasional de altas cantidades (semanal, quincenal o hasta mensual), que no puede definirse como "dependencia" en los términos biomédicos, pero que, como hemos visto, es una pauta cultural difícil de "romper", tanto por su ambivalencia, por las necesidades subjetivo-colectivas que satisface (Cortés, 1988) como por el papel que juega en la configuración de las relaciones sociales.

La incapacidad de beber en solitario, de negarse a dar la "tanda" o de no poder rechazar con facilidad la invitación a beber con alguien cuando se convoca al "tomar dos", junto con lo positivo encontrado en el consumo con "la banda", sugieren la existencia de una dependencia al consumo colectivo de alcohol. Para explicarla me basaré en las ideas de Bateson (1998) sobre la relación sobriedad/intoxicación, y de la cual elaboró una crítica desde un enfoque sistémico que cuestionaba la idea de la "batalla" contra del alcohol y la ilusión de "ganarle". El autor resalta un problema de percepción sobre la relación con esta sustancia, caracterizado por una mirada individual y asistémica.

Propongo entender el consumo colectivo de alcohol, y su dependencia sistémica (Bateson, 1998) o relacional (Menéndez Spina, 2009a) como una práctica social que:

a) Es inherente al sistema de relaciones socioculturales en las que ocurre, por lo que se configura y significa en ellas y no de manera aislada ni en la individualidad.

b) Por lo tanto, y para algunos sujetos, el consumo puede resultar "inevitable", dado su carácter social y relacional.

c) Quien bebe no puede ser totalmente "independiente" o "autónomo" al sistema de relaciones donde se configura esta ingesta colectiva, por lo que el "autocontrol" o la idea de "ganarle" la batalla al alcohol puede ser muy difícil o hasta imposible para algunos sujetos.

d) Sugiere una suerte de "rasgo cultural" que, sobre el consumo de bebidas alcohólicas, se distingue por su negación o extrañamiento respecto a sí mismo/a (o pauta ideal o "formal"), planteando por ello el "problema" del alcohol como ajeno ("borrachos" vs. "tomadores"), pero que en la práctica es un elemento del sistema de relaciones sociales cotidianas de los sujetos y de por lo menos una parte de su subjetividad (pauta real), y que, dicho sea de paso, es una dimensión del consumo -la subjetividad- que amerita mayor investigación local e interdisciplinaria.

${ }^{15}$ INEGI <https: / / www.inegi.org.mx/programas/mortalidad/default.html\#Tabulados>. 
Ahora bien, interesado en establecer un diálogo con la biomedicina, retomo a continuación la revisión histórico-conceptual que realizó Portero Lazcano (2015) sobre la clasificación de los trastornos por consumo de sustancias. El autor señala que el esquema imperante en el sistema taxonómico de la Asociación Americana de Psiquiatría y de la Organización Mundial de la Salud había sido considerar el abuso de alcohol como aquel consumo donde el sujeto no había perdido la "libertad" frente a las drogas, mientras que la dependencia implicaba la pérdida de esta libertad, quedando "esclavizado" ante la sustancia psicoactiva.

Este sistema taxonómico nos remite a los gobiernos de alcohol a los que alude Márquez Osuna (2014), que evocan la noción de libertad y su vinculación con el consumo de alcohol, al encontrar sus orígenes en los movimientos de temperancia apoyados en el positivismo de la época porfirista y en los sistemas morales que intentaron establecer un vínculo entre la voluntad y la libertad, llevando la discusión al campo de la abstinencia y del control de sí mismo.

No obstante, Portero Lazcano (2015) indica que la nueva versión del Manual Diagnóstico y Estadístico de los Trastornos Mentales (DSM5) está transitando -no sin tensiones dentro del propio saber biomédico-de un sistema clasificatorio categórico a uno dimensional, que si bien supone importantes retos prácticos, como en lo relativo a la impartición de justicia, uno de sus aportes es la superación paulatina de un sistema binario de clasificación (de valores presentes o ausentes) a uno dimensional, que reconoce los matices y la complejidad de ciertos cuadros clínicos (Widakovich, 2012).

En otras palabras: desde un análisis categórico, un trastorno por consumo de alcohol en términos biomédicos implicaría identificar si un individuo presenta o no cierto número de criterios diagnósticos (control deficitario, deterioro social, consumo de riesgo y criterios farmacológicos), mientras que desde uno dimensional lo fundamental sería la expresión de estos criterios en un continuum y no en la polaridad presencia/ausencia. En última instancia, "la frontera entre la psicología 'normal' y la psicopatología [es] a veces caprichos[a]" (Widakovich, 2012: 372).

En síntesis, mi llamado a ir más allá de una visión individual y "adictiva" de la dependencia no puede derivar en "patologizar" entonces el consumo colectivo y excesivo de alcohol pues, como se ha mostrado, éste se vive también desde sus atributos positivos; no obstante, no podemos ignorar tampoco que en ciertas circunstancias y relaciones el consumo colectivo deriva en sentidas afectaciones a la salud de los sujetos y conjuntos sociales. Sirva este análisis como una invitación y recordatorio a encontrar nuestros puntos en común entre los saberes médicos, biomédicos y socioculturales.

\section{A modo de conclusiones}

Los procesos de s/e/a-p, como la respuesta ante la pandemia por Covid-19, sacan a la luz las razones y "sinrazones" de todo sistema a nivel histórico, político-económico, cultural e ideológico. La vinculación COVID-19/alcohol no fue la excepción, pues resalta por un lado los remanentes ideológicos del "orden y progreso" de hace un siglo, y confirma por el otro las pautas culturales, ideales y reales que caracterizan el consumo de alcohol en nuestra entidad.

Las prohibiciones alcohólicas gubernamentales de 2020 , las reacciones sociales ante éstas y las cifras de los casos de intoxicación aguda por alcohol así como de contagios confirmados de covid-19 advierten de la urgente necesidad de que las políticas y acciones públicas incorporen en sus análisis la dimensión sociocultural de los procesos de s/e/a-p, no para ignorar lo negativo que conllevan, sino para encarar su complejidad (proceso de alcoholización).

Una de las evidencias etnográficas más recientes corrobora que el consumo de alcohol en Yucatán, o por lo menos de una parte de la población, se representa y se vive como una acción social ambivalente, esto es, que se mueve, mantiene y reproduce a partir del reconocimiento simultáneo de sus efectos negativos y positivos. Esta ambivalencia no debe reducirse a una contradicción entre lo dicho y lo hecho y, en cambio, sí debe conducirnos a una mayor compresión de que los saberes sociales (representaciones y prácticas) pueden expresar estas discontinuidades.

Asimismo, la centralidad del consumo colectivo de alcohol por encima y en contra del consumo solitario revela que la dependencia en términos de adicción individual no nos permite entender por qué hombres y mujeres pueden beber altas cantidades de alcohol cuando están acompañados/as y no así en solitario. Esto nos reta a problematizar la dependencia para mirarla no sólo desde el lente de los criterios patológicos individuales, sino desde las relaciones sociales que localmente han configurado un patrón cultural de consumo caracterizado por su colectividad, sus altas cantidades de consumo por ocasión y su ambivalencia.

Para cerrar, considero que uno de los retos que enfrentan los estudios socioculturales contemporáneos de los procesos de s/e/a-p es dar cuenta tanto de las experiencias y discursos de los sujetos sociales como de sus prácticas, de lo que de hecho hacen, para ser 
capaces de analizar las continuidades y discontinuidades que suelen distinguir a estos complejos procesos.

\section{Fuentes}

Barguet loeza, Daniel

2020 "Por 'Ley seca', hacen compras de pánico", en Milenio, 14 de julio de 2020 < https: / / www milenio.com/estados/ley-seca-coronavirusgenera-compras-panico-yucatam $>$ [20 de septiembre de 2020].

Bateson, Gregory

1998 Pasos hacia una ecología de la mente, LohleLumen, Buenos Aires, 552 pp.

Bourdieu, Pierre, Jean-Claude Chamboredon

y JeAn-Claude Passeron

2002 El oficio de sociólogo, Siglo xxi Editores, México, $376 \mathrm{pp}$.

CORTÉs, BEATRIZ

1988 "La funcionalidad contradictoria del consumo colectivo de alcohol", en Nueva Antropología, año 10, núm. 34, pp. 157-185.

DiARIO DE YUCATÁN

2020 "Más detenidos por venta clandestina de cervezas en Mérida”, en Diario de Yucatán, 11 de abril <https://www.yucatan.com.mx/merida/ mas-detenidos-por-venta-clandestina-decervezas-en-merida> [ 18 de junio de 2020].

Gilbert, JeAN

1985 "Mexican-americans in California: Intracultural variation in attitudes and behavior related to alcohol", en Linda Bennett y Genevieve Ames (eds.), The American Experience with Alcohol: Contrasting Cultural Perspectives, Plenum Press, Nueva York, pp. 255-277.

Heath, Dwight B.

2012 Drinking Occasion. Comparative Perspectives on Alcohol and Culture, Routledge, Londres, $240 \mathrm{pp}$.

LA VERDAD

2020 “'Ley Seca' en Yucatán provoca compras de pánico; todos olvidaron la cuarentena", en $L a$ Verdad, 10 de abril <https: / / laverdadnoticias. com / yucatan / Ley-Seca-en-Yucatan provoca-compras-de-panico-todos-olvidaronla-cuarentena-20200410-0028.html> [18 de junio de 2020].

LiNTON, RALPH

1945 Cultura y personalidad, Fondo de Cultura Económica, México, 155 pp.

Márguez Osuna, AngélicA

2014 "Las políticas sobre el consumo de alcohol: Ciencia, orden y voluntad. Mérida, Yucatán. Siglos xix y xx", tesis de maestría, Facultad de Antropología-Universidad Autónoma de Yucatán, Mérida, 154 pp.

Medina-Mora, María Elena,

SHOSHANA BERENZON

y Guillermina Natera Rey

1999 "El papel del alcoholismo en las violencias", en Gaceta Médica Mexicana, vol. 135, núm. 3, pp. 282-287 <http://www.anmm.org. mx/ bgmm/1864_2007/1999-135-3-282-287. pdf> [enero de 2019].

Menéndez Spina, Eduardo Luis

2009a De sujetos, saberes y estructuras. Introducción al enfoque relacional en el estudio de la sa- lud colectiva, Lugar Editorial, Buenos Aires, $312 \mathrm{pp}$.

Menéndez Spina, Eduardo Luis

2009b "De racismos, esterilizaciones y algunos otros olvidos de la antropología y la epidemiología mexicanas", en Salud Colectiva, vol. 5, en $<$ http: / / revistas.unla.edu.ar/salud colectiva/article/view/258 > DOI: 10.18294/sc. 2009.258

Menéndez Spina, Eduardo Luis

2015 "Las enfermedades ¿son solo padecimientos?: biomedicina, formas de atención 'paralelas' y proyectos de poder", en Salud Colectiva, vol. 11, núm, 3, pp. 301-330.

Menéndez SPina, EduARdo Luis

2020 Morir de alcohol: Saber y hegemonía médica. Nueva edición corregida y aumentada, Universidad Nacional de Lanús, Buenos Aires. <http: / / isco.unla.edu.ar/edunlcuadernos / catalog/book/8> [febrero de 2020].

Menéndez, Eduardo y René Di Pardo

1996 De algunos alcoholismos y algunos saberes, Centro de Investigaciones y Estudios Superiores en Antropología Social, México.

Miranda, Justino

2020 "La otra epidemia en México: morir por alcohol adulterado", en El Universal, 20 de mayo <https: / / www.eluniversal.com.mx/estados / la-otra-epidemia-en-mexico-morir-poralcohol-adulterado> [ 18 de junio de 2020].

Moreno Cabrera, Sergio Andrés

2019 "Entre 'saber tomar' y 'no saber tomar': consumo de alcohol y consecuencias positivas, negativas y ambivalentes en la salud, recursos económicos y relaciones sociales de varones y mujeres de un municipio de Yucatán", tesis doctoral, Centro de Investigaciones y Estudios Superiores en Antropología Social, Ciudad de México, 555 pp. <http://ciesas. repositorioinstitucional.mx/jspui/handle/ $1015 / 959>$

Moreno Cabrera, Sergio Andrés

2020a "Entre "saber y no saber tomar": representaciones y prácticas de varones y mujeres sobre el consumo de alcohol en Yucatán”, en Salud Colectiva, vol. 16 <http://revistas.unla.edu. ar/saludcolectiva/article/view/2533/1637> DOI: $10.18294 / \mathrm{sc} .2020 .2533$ [ 12 de agosto de 2020].

Moreno Cabrera, Sergio Andrés

2020b "El consumo de alcohol como "chivo expiatorio' de las violencias cotidianas de género", ponencia presentada en Simposio del VI Congreso Asociación Latinoamericana de Antropología, llevado a cabo en Uruguay [modalidad virtual, noviembre].

Moreno Cabrera, Sergio Andrés

En prensa "Entre la dominación y la vulnerabilidad: poder, padecer y privilegios masculinos: El caso del consumo de alcohol en las relaciones sociales cotidianas", en Edith Yesenia Peña Sánchez y Lilia Hernández Albarrán (coords.), Cuerpos, géneros y sexualidades. Contextos mesoamericanos y contemporáneos, Instituto Nacional de Antropología e Historia, México.

\section{Portero Lazcano, Guillermo}

2015 "DSM-5. Trastornos por consumo de sustancias. ¿Son problemáticos los nuevos cambios en el ámbito forense?", en Cuadernos 
de Medicina Forense, vol. 21, núm. 3-4, pp. 96-104 <http://scielo.isciii.es/scielo.php?script=sci_abstract\&pid=S1 135-76062015000

RICO, DAVID $200002>$ [ 8 de agosto de 2020].

2020

"Clandestinos aprovechan Ley Seca para vender alcohol a sobreprecio en Yucatán”, en Por Esto, 17 de agosto <https://www.poresto. net/yucatan / 2020/8/17/clandestinos aprovechan-ley-seca-para-vender-alcoholsobreprecio-en-yucatan-5924.html> [20 de septiembre de 2020].

RodríGuez GonzÁlez, León AdÁN

2015 "Intoxicación aguda por alcohol. Guía de certificación médica del ejército mexicano", en $R e$ vista Sanidad Militar México, vol. 69, núm. 5, pp. 467-475 <http: / /omextad.salud.gob.mx/ contenidos/noticias/intoxicacion_aguda_ alcohol.pdf $>$ [20 de septiembre de 2020].

Ronzani, Telmo Mota y Graciela Touzé

2020 "Consumos de sustancias psicoactivas: del castigo al cuidado", en Salud Colectiva, vol. 16. DOI: $10.18294 /$ sc.2020.3100
Villatoro-Velázguez, Jorge A. et al.

2017 Encuesta Nacional de Consumo de Drogas, Alcohol y Tabaco 2016-2017: Reporte de Alcohol, Instituto Nacional de Psiquiatría Ramón de la Fuente Muñiz/Instituto Nacional de Salud Pública/Comisión Nacional Contra las Adicciones/Secretaría de Salud, México $<$ www.inprf.gob.mx> [ 11 de enero de 2018].

Widakovich, Christian

2012 "El enfoque dimensional vs el enfoque categórico en psiquiatría: aspectos históricos y epistemólogicos”, en Alcmeon Revista Argentina de Clínica Neuropsiquiátrica, vol. 17, núm. 4, septiembre, pp. 365-374 <https://www. alcmeon.com.ar/17/68/06 Bidacovich.pdf> [8 de agosto de 2020].

Yucatán Ahora

2020 "Dos muertos más por alcohol adulterado: ya son 16 defunciones en esta ley seca", $\mathrm{Yu}$ catán Ahora, 22 de mayo <https://yucatan ahora.mx/dos-muertos-mas-por-alcoholadulterado-ya-son-16-defunciones-en-estaley-seca/> [20 de septiembre de 2020]. 